\title{
THE THREEHUNDRED: the structure and properties of cosmic filaments in the outskirts of galaxy clusters
}

\author{
Agustin Rost ${ }^{\circledR}, 1,2 \star$ Ulrike Kuchner ${ }^{\circledR}, 2$ Charlotte Welker, ${ }^{3,4}$ Frazer Pearce, ${ }^{2}$ Federico Stasyszyn ${ }^{(\bullet)},{ }^{1}$

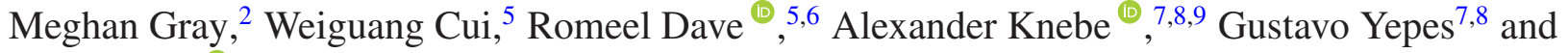 \\ Elena Rasia ${ }^{\circledR 10}$ \\ ${ }^{1}$ Instituto de Astronomía Teórica y Experimental (IATE), Consejo Nacional de Investigaciones Científicas y Técnicas, Laprida 854, Córdoba X5000BGR, \\ Argentina \\ ${ }^{2}$ School of Physics, Astronomy, University of Nottingham, Nottingham NG7 2RD, UK \\ ${ }^{3}$ Department of physics and Astronomy, Krieger School of Arts and Science, The Johns Hopkins University, Baltimore MD 21218, USA \\ ${ }^{4}$ Department of Physics and Astronomy, McMaster University, Ontario L8S 4K1, Canada \\ ${ }^{5}$ Institute for Astronomy, University of Edinburgh, Royal Observatory, Edinburgh EH9 3HJ, UK \\ ${ }^{6}$ Department of Physics and Astronomy, University of the Western Cape, Bellville, Cape Town 7535, South Africa \\ ${ }^{7}$ Departamento de Física Teórica, Módulo 15, Facultad de Ciencias, Universidad Autónoma de Madrid, E-28049 Madrid, Spain \\ ${ }^{8}$ Centro de Investigación Avanzada en Física Fundamental (CIAFF), Universidad Autónoma de Madrid, E-28049 Madrid, Spain \\ ${ }^{9}$ International Centre for Radio Astronomy Research, The University of Western Australia, 35 Stirling Highway, Crawley, WA 6009, Australia \\ ${ }^{10}$ INAF, Osservatorio Astronomico di Trieste, via Tiepolo 11, I-34131 Trieste, Italy
}

Accepted 2020 December 3. Received 2020 December 3; in original form 2020 October 9

\begin{abstract}
Galaxy cluster outskirts are described by complex velocity fields induced by diffuse material collapsing towards filaments, gas, and galaxies falling into clusters, and gas shock processes triggered by substructures. A simple scenario that describes the large-scale tidal fields of the cosmic web is not able to fully account for this variety, nor for the differences between gas and collisionless dark matter. We have studied the filamentary structure in zoom-in resimulations centred on 324 clusters from THE THREEHUNDRED project, focusing on differences between dark and baryonic matter. This paper describes the properties of filaments around clusters out to five $R_{200}$, based on the diffuse filament medium where haloes had been removed. For this, we stack the remaining particles of all simulated volumes to calculate the average profiles of dark matter and gas filaments. We find that filaments increase their thickness closer to nodes and detect signatures of gas turbulence at a distance of $\sim 2 \mathrm{~h}^{-1} \mathrm{Mpc}$ from the cluster. These are absent in dark matter. Both gas and dark matter collapse towards filament spines at a rate of $\sim 200 \mathrm{~km} \mathrm{~s}^{-1} \mathrm{~h}^{-1}$. We see that gas preferentially enters the cluster as part of filaments, and leaves the cluster centre outside filaments. We further see evidence for an accretion shock just outside the cluster. For dark matter, this preference is less obvious. We argue that this difference is related to the turbulent environment. This indicates that filaments act as highways to fuel the inner regions of clusters with gas and galaxies.
\end{abstract}

Key words: galaxies: clusters: general-galaxies: clusters: intracluster medium-galaxies: haloes.

\section{INTRODUCTION}

Matter in the Universe is structured as a complex network called the cosmic web (Bond, Kofman \& Pogosyan 1996). In this picture, galaxy clusters mark the high-density nodes of the network, connected by a multitude of elongated filaments and walls that, together, host most of the mass in the Universe. In contrast, cosmic voids occupy the majority of the volume, but are relatively depleted of material (Forero-Romero et al. 2009; Cautun et al. 2014; Tempel et al. 2014). Cosmological simulations of the formation of this largescale structure suggest a sequence in which dark matter and gas are gravitationally attracted from underdense regions (3D voids) to increasingly denser regions of $2 \mathrm{D}$ walls, which contract into $1 \mathrm{D}$

^E-mail: a_rost@unc.edu.ar filaments through which the material flows attracted by the large potential well of (clusters located in) the nodes. The underlying physics of this structure formation is based on theoretical work by Zel'dovich (1970) who recognized the role of the large-scale tidal field as the major driving force in shaping the cosmic web, based on the initial density perturbations in the early Universe (Arnold, Shandarin \& Zeldovich 1982; Shandarin \& Klypin 1984; Gurbatov, Saichev \& Shandarin 1989; Shandarin \& Zeldovich 1989; Hidding, Shandarin \& van de Weygaert 2014).

Today, approximately 40 per cent of the baryons in the Universe are expected to consist of gas at temperatures of $10^{5}-10^{7} \mathrm{~K}$, the warm hot intergalactic medium (WHIM), which is investigated as a possible solution of the so-called missing baryon problem (Cen \& Ostriker 1999; Davé et al. 2001; Reimers 2002; Bykov, Paerels \& Petrosian 2008). To a large extent, the WHIM is found in filaments, thus representing an important observational tracer of the cosmic 
web (e.g. Cui et al. 2019; Umehata et al. 2019). Indeed, the baryon fraction of filaments is found to be higher than that of galaxies (however, slightly lower than that of the cosmic mean) and with gas temperatures uniform as a function of the distance to the filament (Gheller \& Vazza 2019). However, unlike the hot gas accumulating in the gravitational wells of clusters, the WHIM is much more challenging to detect. Recent promising results have lately pushed the study of the cosmic web into the focus of galaxy formation theory, simulation, and observations alike (Nicastro et al. 2013; Eckert et al. 2015; Bonamente et al. 2016; Cui et al. 2018a; Tanimura et al. 2019, 2020). Simulation-based studies have shown that filament properties, such as the average gas temperature, mass, volume, and radius, follow scaling relations (e.g. Gheller et al. 2015). However, since direct detections often rely on stacking the signal of multiple objects (e.g. Tanimura et al. 2019, 2020), a better understanding of the distribution of the WHIM could help improve its detection in future observational studies.

Cosmological filaments cover a variety of properties ranging from thin tendrils pervading voids to thick bridges between cluster pairs that boast many substructures and collapsed haloes, thus playing an important role of transporting matter into galaxy clusters (e.g. Cautun et al. 2014; Kraljic et al. 2018). A number of studies using simulations and observations have used cosmic filaments to characterize anisotropic accretion to further establish a picture of steady filament dynamics in less and intermediate dense regions (Pichon et al. 2011; Codis et al. 2012; Tempel, Stoica \& Saar 2013; Codis, Pichon \& Pogosyan 2015; Laigle et al. 2015; Libeskind et al. 2015; Kraljic et al. 2019). The multiscale and diffuse nature of filaments makes defining and distinguishing between different structures a non-trivial task. To add to the difficulty, different filament-finding algorithms are based on different implicit definitions of these objects. This leads to wide-ranging discrepancies in relation to the nature of identified objects found by the different filament finders (Libeskind et al. 2018; Rost et al. 2020), including DISPERSE (Sousbie 2011), Semita (Pereyra et al. 2019), Nexus (Aragón-Calvo et al. 2007; Cautun, van de Weygaert \& Jones 2013), or Bisous (Tempel et al. 2014). Despite these drawbacks, understanding the large-scale structures of the Universe, and how the galaxies they host evolve, remain key science challenges.

Most current research has been focusing on large-scale filaments of dozens of Mpc in length and over large volumes of the Universe (e.g. Tempel et al. 2013; Codis et al. 2015; Laigle et al. 2015; Malavasi et al. 2017; Kraljic et al. 2018, 2019; Ganeshaiah Veena et al. 2019; Cui et al. 2018a, 2019). These investigations have established that cosmological filaments are crucial structures for the dynamics and properties of gas and galaxies alike (e.g. Codis et al. 2012; Cautun et al. 2014). The filaments are surrounded by complex velocity fields that, to a first order, can be explained by the Zel'dovich approximation (Zel'dovich 1970). This assumption leads to the expectation of a laminar flux towards the filamentary axis, a manifestation of diffuse material collapsing into filaments along two axes. However, this simple approach does not account for differences between gas and collisionless dark matter or for the dynamics induced by local cosmic web components, like galaxies falling into clusters.

Both observations and simulations have rarely included the special case of filaments around massive galaxy clusters - dense areas of highly mixed and turbulent environments, where gas undergoes significant shocks (Paul et al 2011; Power et al. 2020). Likely, the properties of gas and dark matter in cluster filaments therefore differ to that found in filaments in the more general large-scale cosmic web. In particular, there is evidence of gas shock processes triggered by the filamentary substructure falling into clusters that could lead to turbulent motion of the gas outside them (Power et al. 2020). Near a filament's spine, cold gas may be expected to flow more smoothly, transporting relatively cold gas into the clusters. This is analogous to the cold flows that are found to shape forming galaxies at high redshifts (Birnboim \& Dekel 2003; Dekel et al. 2009; Pichon et al. 2011; Danovich et al. 2012; Dubois et al. 2012; Cornuault et al. 2018). These considerations raise questions like how exactly is gas accreted towards clusters? Is filament accretion more efficient than a purely gravitational dark matter infall?

As the sites of possible pre-processing and matter accretion on to clusters, the study of filaments in cluster outskirts is now gaining momentum as simulations and observations have improved (see Walker et al. 2019, for a recent summary). The outskirts of galaxy clusters represent ideal laboratories to study both matter build-up in the Universe and galaxy evolution, as they are convergence points for filamentary networks and offer scenarios of phenomena involving high-density nodes, intermediate density filaments, and low density 'field' regions. In this paper, we address this specific case and study the gas and dark matter properties of filaments in the vicinity of massive galaxy clusters. We view this analysis of filaments in highdensity environments as an extension to similar studies that focused on filaments in a wider mix of environments, and on a wider range of scales. We investigate the structure and properties of filaments through their diffuse gas surrounding haloes - closely related to the WHIM - as well as the underlying dark matter.

The paper is structured as follows: In Section 2, we detail the simulations of 324 massive cluster our study is based on and the identification of cluster filaments based on gas particles. In Section 3, we investigate the interplay between haloes and filaments and study parameters such as filament length, density, and temperature. We also study the role of the clusters that the filaments are connected to and define a characteristic thickness based on density profiles. In Section 4 , we discuss the dynamics of gas and dark matter around the cluster and disentangle flows towards the cluster and towards and along filaments before summarizing our conclusions in Section 5. In this paper, we are using Planck cosmology with $\Omega_{\mathrm{M}}=0.307, \Omega_{\mathrm{B}}=$ $0.048, \Omega_{\Lambda}=0.693, h=0.678, \sigma_{8}=0.823$, and $n_{s}=0.96$.

\section{DATA AND FILAMENT FINDING}

\subsection{THE THREEHUNDRED simulations}

In this work, we use 324 cluster zoom-in simulations at $z=0$ of THE THREEHUNDRED project ${ }^{1}$ (Cui et al. 2018b). The sample was built form a mass-complete sample of clusters by choosing the 324 most massive virialized objects at $z=0$ within the MultiDark2 simulation volume (Klypin et al. 2016), identified with the ROCKSTAR halo finder (Behroozi, Wechsler \& Wu 2013). The cluster masses range from $M_{200}=6.08 \times 10^{14} h^{-1} \mathrm{M}_{\odot}$ to $M_{200}=2.62 \times 10^{15} h^{-1} \mathrm{M}_{\odot}$. Particles in these clusters were then traced back to their initial positions and resimulated using the full-physics code GADGET-X (Springel 2005; Murante et al. 2010; Rasia et al. 2015; Beck et al. 2016), which uses a modern Lagrangian smoothed particle hydrodynamics (SPH) approach. The high-resolution volumes are spherical regions of radius $15 h^{-1} \mathrm{Mpc}$ centred on each cluster and contain dark matter and gas particles with initial masses of $12.7 \times 10^{8} h^{-1} \mathrm{M}_{\odot}$ and $2.36 \times 10^{8} h^{-1} \mathrm{M}_{\odot}$, respectively. This usually corresponds to an extent of radius $5 R_{200}$ at $z=0$. Time-steps between redshifts $z \sim$ 17 and $z=0$ are stored in 129 snapshots and are available for a

\footnotetext{
${ }^{1}$ https://the300-project.org
} 
plethora of studies that trace orbits and cluster mass build-up over time (Arthur et al. 2019; Haggar et al. 2020).

Haloes and subhaloes were identified in each snapshot using the $\mathrm{AHF}^{2}$ halo finder (Amiga's Halo Finder, Knollmann \& Knebe 2009). AHF accounts for dark matter, gas, and stars and returns properties that define each halo, such as luminosities, stellar masses, and angular momentum among others. The sizes and masses of haloes used in our analysis refer to $R_{200}$ and $M_{200}$, where $R_{200}$ is the radius of a sphere enclosing an overdensity of 200 times the critical density of the Universe at $z=0$. For this work, we only consider haloes with masses above $10^{11} h^{-1} \mathrm{M}_{\odot}$ (which corresponds to around 80 dark matter particles) and haloes closer than $15 h^{-1} \mathrm{Mpc}$ from the centre of the Lagrangian/re-simulated region, at redshift zero.

For the simulation environment we are studying in this paper, i.e. largely a low-overdensity regime, it is important to recognise that Lagrangian particle-based methods such as SPH are not well suited to recover sharp discontinuities such as shocks due to the enforced relatively long search lengths required to find a suitable number of particle neighbours. Adaptive mesh or moving mesh based methods would be better able to resolve these. As such we need to be careful with our analysis and bear this in mind when discussing our results. Effectively, small-scale structures cannot be readily resolved within the low-density material. For this reason, we generally stack our data, a process that would naturally smooth these structures anyway. Further, we do not employ the recovered SPH gas density from GADGET-X but rather recalculate density from the stacked particle locations. Lowering our particle mass and so improving our ability to resolve sharp discontinuities would improve our ability to see small structures but any such effort is limited because a factor of 2 decrease in linear scale requires nearly an order of magnitude increase in particle number. We note that the size of the structures discussed below is significantly above our resolution limit in this sense and as such they are well resolved.

\subsection{Filament extraction based on gas particles}

We identify filaments with the filament finder DISPERSE (Sousbie 2011), a tool designed to extract multiscale structures. From a given distribution of tracers, DISPERSE identifies significant topological features in the density field (maxima, minima, and saddle points) as well as ridges that connect them. In short, DISPERSE provides the positions and identity of a number of web-like structural features where the filaments themselves are the lines connecting the singularities, defined by the gradient field (Sousbie 2011; Libeskind et al. 2018). In this context, nodes can be thought as an extreme point where DM haloes reside and saddle points are next extreme point along the filament axis.

For our analysis, we extract filaments based on the 3D number density of gas particles in each simulation box. The gas density was first binned in a 3D grid of $30 \mathrm{~h}^{-1} \mathrm{Mpc}$ width and pixel resolution of $150 \mathrm{kpc} \mathrm{h}^{-1}$ on each side. This grid was then smoothed over eight pixels with a Gaussian kernel. This process allows us to focus on large cosmic filaments that connect groups and cluster centres, and excludes thin tendrils. DISPERSE then identified filaments using an absolute persistence cut-off 0.2 (corresponding roughly to a $5 \sigma$ persistence threshold using haloes). The persistence is defined as the ratio of the density value of a pair of critical points (e.g. node and saddle point) and governs the robustness of the feature and is used to additionally filter out low significant filaments.

\footnotetext{
${ }^{2}$ http://popia.ft.uam.es/AHF
}

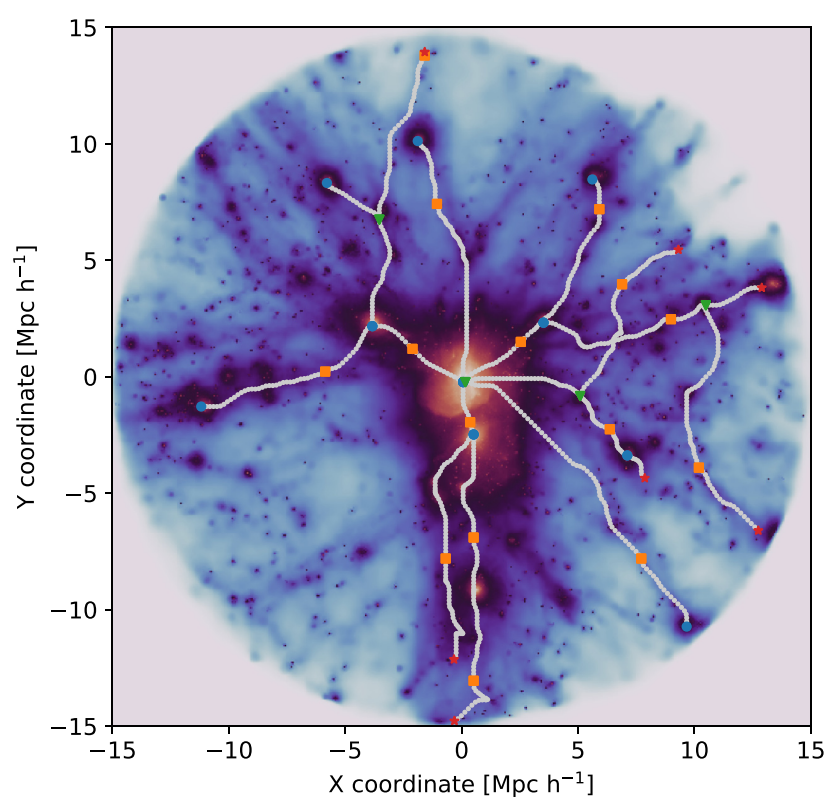

Figure 1. One example cluster of THE THREEHUNDRED simulation suite with its extracted filament network based on gas particles. The background shading indicates projected gas particle density along the $z$-axis of the cluster, rendered with SPH-viewer (Benitez-Llambay 2015). The recovered DISPERSE filamentary network, which was identified using the 3D distribution, is shown in white, symbols indicate different features of the filamentary network. The blue circles: nodes, the orange squares: saddle points, the inverted green triangles: bifurcations, and the red stars: exit points.

DISPERSE returns the identified ridges as a set of segments that construct the persistent skeleton of the network as well as the topologically robust extrema, i.e. saddle points and nodes. Fig. 1 shows the filament network of Cluster 1 (plotted in white on top of the gas density in blue shading) as an example. In addition to nodes (the blue points) and saddle points (the orange squares), we also show bifurcation points that mark the location where a filament splits (the green triangles). Often, bifurcation points indicate local peaks in the density field, however, they are deemed less significant than nodes. The red stars indicate exit points: this is the location where a filament leaves the extent of the simulated zoom region around the cluster. Combining all networks in 324 zoom regions, we thus identify a sample of 11058 filaments based on gas particles. While the example shown in Fig. 1 offers an obvious clue that the identified network indeed traces the underlying structure of the density field, we refer the reader to Kuchner et al. (2020), in which detailed tests of the filament extraction method on gas and mock galaxies in the same simulations, as well as comparisons between detections in 2D and $3 \mathrm{D}$ are presented.

In this work, we define a filament as a structure that connects a topological peak (node) with a saddle point, sometimes splitting along the way into multiple branches at bifurcation points. Thus, any single node controls all filaments connected to that node as far as the first saddle points. Naturally, filaments continue beyond the saddle points, but they are then controlled by a different node. As a basic principle, material in filaments on either side of the saddle point will eventually fall into a different halo. This can be thought of as similar to the way water on either side of a watershed may end up in different oceans. Note that this physically motivated definition of filaments may differ from other definitions in the literature that often 
define a filament as joining two nodes. Several filaments together constitute a filament network which is the basis for our analysis.

\section{THE INTERPLAY BETWEEN HALOES AND FILAMENTS}

Filaments are regions of moderate collapse hosting both diffuse material and already bound haloes. These components interact dynamically with one another through accretion, outflows, and stripping. To some extent, halo gas, intrahalo gas, and filament gas mix, thus making the study of the pure diffuse filament gas a challenging task. We approach this challenge by isolating the gas in filaments from the contribution of embedded haloes. This is not easy in the best of cases, and it is important to note that this exercise is complicated by resolution: every simulation's implementation of gas density, temperature, and cooling model governs how well pure spine flows and haloes-outskirt particles can be separated. The exclusion of the halo contribution therefore always represents a compromise.

In this section, we first look at the contribution of the main halo ${ }^{3}$ on the lengths of filaments connected to this halo (Section 3.1), before examining the removal of haloes in filaments - which can represent the main cluster, groups, and individual galaxies - in more depth (Section 3.2). Finally, we determine (Section 3.3) and characterize (Section 3.4) filament profiles based on gas and dark matter densities and contrast the contributions of nodes and distances to the nodes and filaments on these profiles (Section 3.5).

\subsection{The dependence of filament length on the proximity to the central node}

Processes that give rise to filament properties are to a high degree scale-invariant leading to the multiscale nature of filaments. The choices we make during the filament extraction therefore governs the range of scales we are able to investigate. In this study, we choose to focus on cluster filaments, i.e. prominent filaments in outskirts of clusters with $M_{*}>10^{14} \mathrm{M}_{\odot} \mathrm{h}^{-1}$, and have adapted the background density and smoothing accordingly.

To introduce the scales of filaments we are studying in this paper, Fig. 2 shows a histogram of the lengths of all recovered filaments. These filaments have a wide range of lengths, with a small number extending from the centre of the cluster all the way to the edge of the re-simulated region. Naturally, intergroup filaments are on average shorter than intercluster filaments. We distinguish between filaments that are connected to the central halo (shown in red), which we call the central network, and all other filaments. For this case of THE THREEHUNDRED simulations, filaments connected to the central cluster are around $6 h^{-1} \mathrm{Mpc}$ long, while those originating anywhere in the volume are on average about half this length. Of course, these numbers are influenced by the limited volume we are probing in this study.

Other studies such as Malavasi et al. (2020), Tanimura et al. (2020), Gheller et al. (2015), and Gheller \& Vazza (2019) focus on long filaments of dozens of Mpc, whereas others like Kraljic et al. (2019) focus on filaments in the range 3-12Mpc. Here, we report the distance between a node and a saddle point, the length of filaments from one node to the other will typically be twice this value as normally both halves of a bridge between two nodes will be counted separately. Although the length range is not comparable to other

\footnotetext{
${ }^{3}$ In THE THREEHUNDRED simulations, the mass of the central halo in each simulation box is comparable to the entire cluster's mass itself and therefore influences the surrounding cosmic web significantly.
}

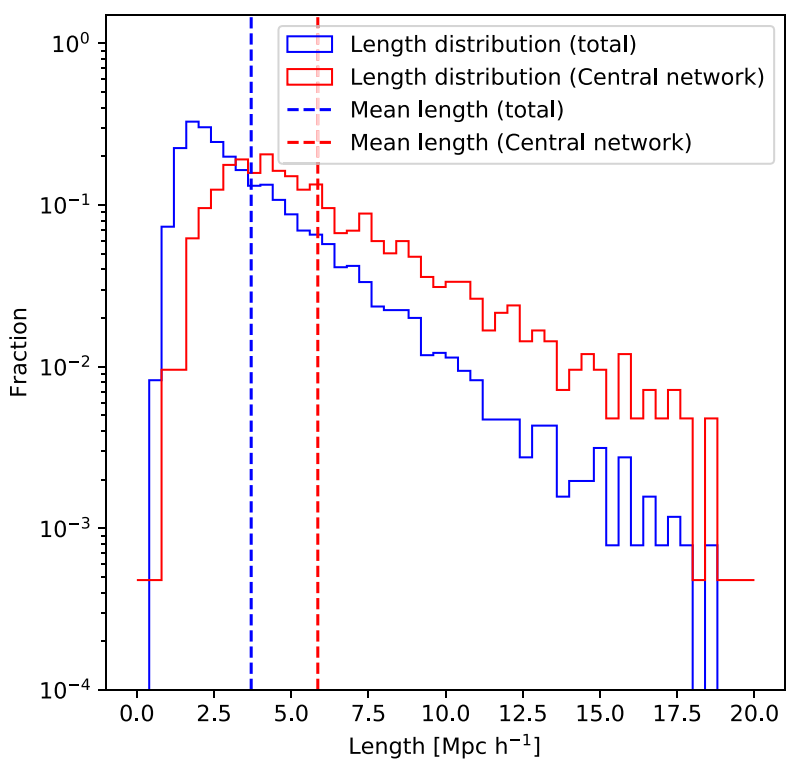

Figure 2. The length distribution of filaments in our sample of 324 clusters is longer for filaments associated with central nodes. Only filaments with a node and a saddle point were considered, those with bifurcation or exit points were excluded. The blue distribution shows that the average length is approximately $3.70 h^{-1} \mathrm{Mpc}$ for the total sample and $5.88 h^{-1} \mathrm{Mpc}$ for filaments connected to the central cluster (shown in red). Filaments run from a node to a saddle point and at least two are needed to bridge between haloes.

studies mentioned above, the trend of having a lower population for longer filaments seen here is widely observed in the literature (Bond, Strauss \& Cen 2010; Galárraga-Espinosa et al. 2020; Malavasi et al. 2020; Rost et al. 2020). Note also that the recovered length and number of filaments depend on the persistence level chosen during filament extraction, with a lower level leading to more nodes and a finer network. As mentioned in Section 2.2, we have deliberately chosen a procedure to robustly extract the main branches of the network that omits small tendrils.

\subsection{The contribution of haloes in filaments}

Haloes are an integral part of filaments. Our aim in this paper is to investigate filament profiles based on the diffuse material of filaments, i.e. without the contribution of haloes. To achieve this, we remove the gas that can be attributed to haloes. A precise and complete removal is, however, unattainable due to the impossibility of clearly determine the boundaries of haloes. We therefore approximate the process by removing material inside spheres around haloes - detailed in this section - leaving only diffuse material.

First, we need to find the appropriate size of the region of halo influence. To do so, we exclude particles within increasingly larger regions around increasingly more massive haloes. Specifically, in this test we exclude particles within $0,1,2$, and 3 times $R_{200}$ (see Figs 3 and 4) and around haloes with log halo masses greater than 10.47, 11.71, 12.94, and $14.18 h^{-1} \mathrm{M}_{\odot}$ (see Fig. 5). We first detail the effect of varying sizes of regions before looking at identifying the appropriate halo mass limit.

To illustrate the removal process, Fig. 3 shows a volume rendered example filament in both gas and dark matter with successively larger excision radii from top to bottom. Removing a volume of $1 \times R_{200}$ around each halo leaves a high-density shell around the centres of some haloes. By removing particles within $3 \times R_{200}$ of haloes, we 

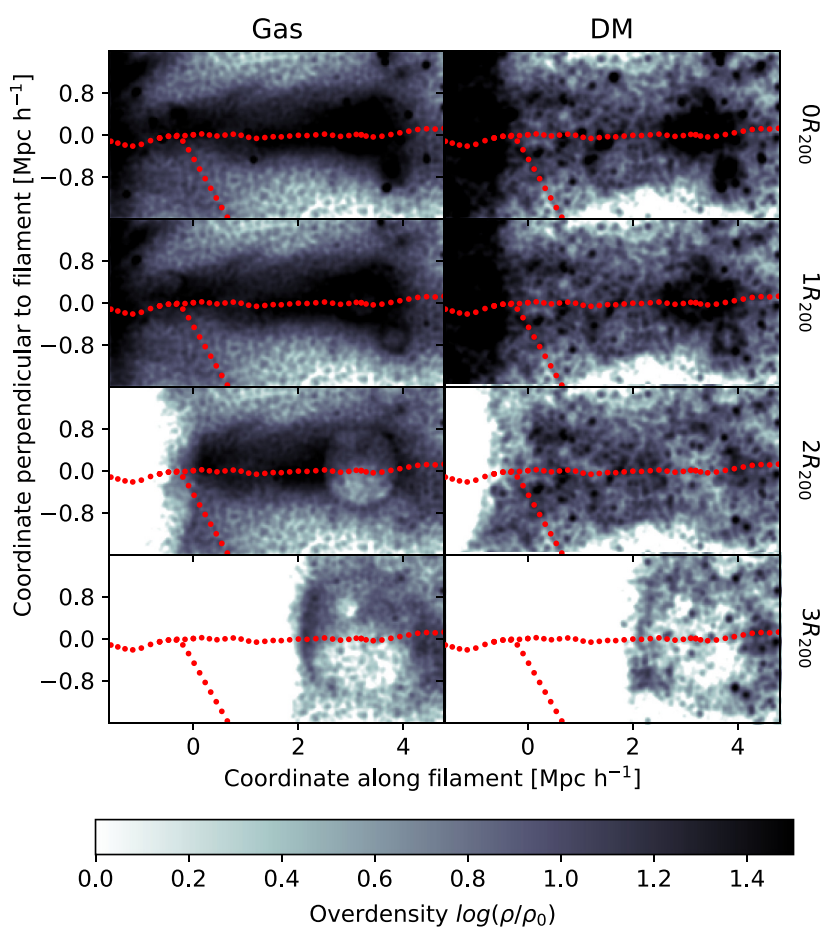

Figure 3. Illustration of the process of isolating diffuse filament material by removing increasingly larger regions around haloes: Column-integrated overdensity of regions surrounding filament 4 of cluster 2. From top to bottom, overdensities after removing $0,1,2$, and 3 times $R_{200}$, respectively. On the left: gas particles. On the right: dark matter particles. DISPERSE filaments are marked in red, note that the central cluster is located to the left side.

risk excluding filament gas. Thus, exciting volumes equivalent to 2 $\times R_{200}$ seems like the best compromise.

Fig. 4 shows an alternative view of the contribution of halo gas and the removal process. The figure shows the density - temperature phase space diagram for all the particles found within the central $10 \mathrm{~h}^{-1} \mathrm{Mpc}$ of the largest cluster in the sample (the same as shown in Fig. 1). The diffuse material of filaments is highly related to the WHIM, as its gas is expected to be distributed along the cosmic web, surrounding galaxies embedded in filaments. Fig. 1) highlights the thermodynamical properties that characterize both haloes and the diffuse filament material. In the four panels, we progressively remove larger volumes surrounding each halo as before. Some of the particles lie within the central hot cluster halo with temperatures around $10^{8} \mathrm{~K}$ - these identify the diffuse intracluster medium while others are gas at high overdensity and temperatures close to $10^{4} \mathrm{~K}$, these are the multiphase particles with a significant cold fraction that represents the reservoir for the star-forming process. As successively larger regions around each of the identified haloes are excised $\left(1,2\right.$, and 3 times $R_{200}$, respectively, as indicated in the panels), we see both this hot halo gas and the galactic phase largely disappearing. Removing particles out to $1 \times R_{200}$ (top righthand panel in Fig. 4) leaves a considerable amount of warm-diffuse particles. By increasing the limiting radius in units of $R_{200}$, the maximum density decreases significantly and both the hot and starforming phases are largely excluded. The bottom two panels indicate that essentially only underdense material and WHIM remain once regions inside twice $R_{200}$ are removed. After removing the effect of haloes, the temperature and density distributions remain stable along the filament spine. We only see a small increase of the gas temperature

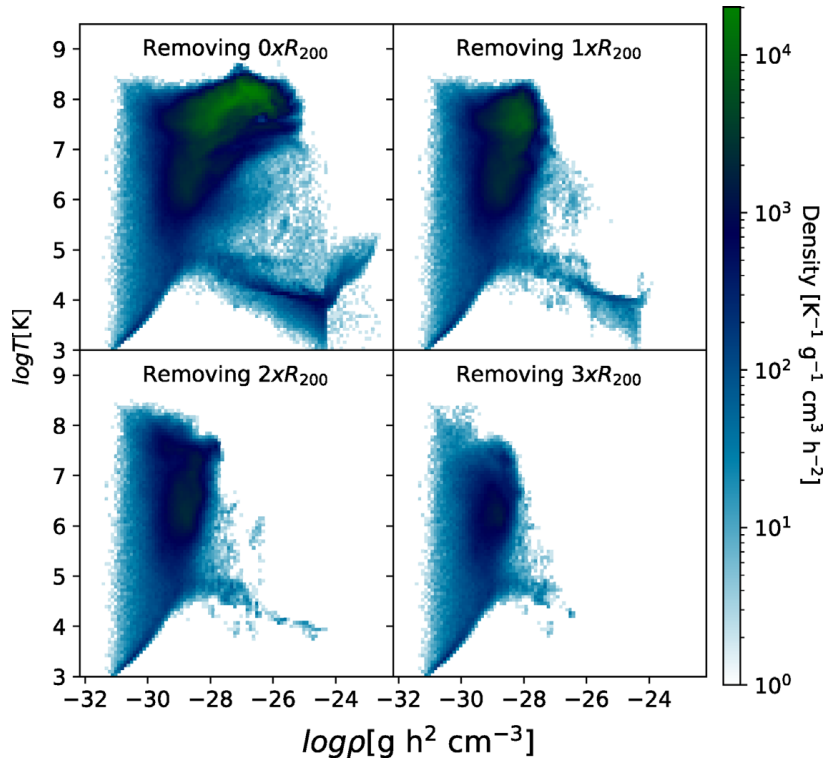

Figure 4. Demonstration of the contribution of haloes on the density temperature phase-space diagram of gas particles contained within the filaments of cluster 0001. The top left-hand panel displays all the particles, while in the other panels successively larger regions around each halo of which mass limit, $10^{11}$ are excised as indicated, the colour represents the value of the distribution function.

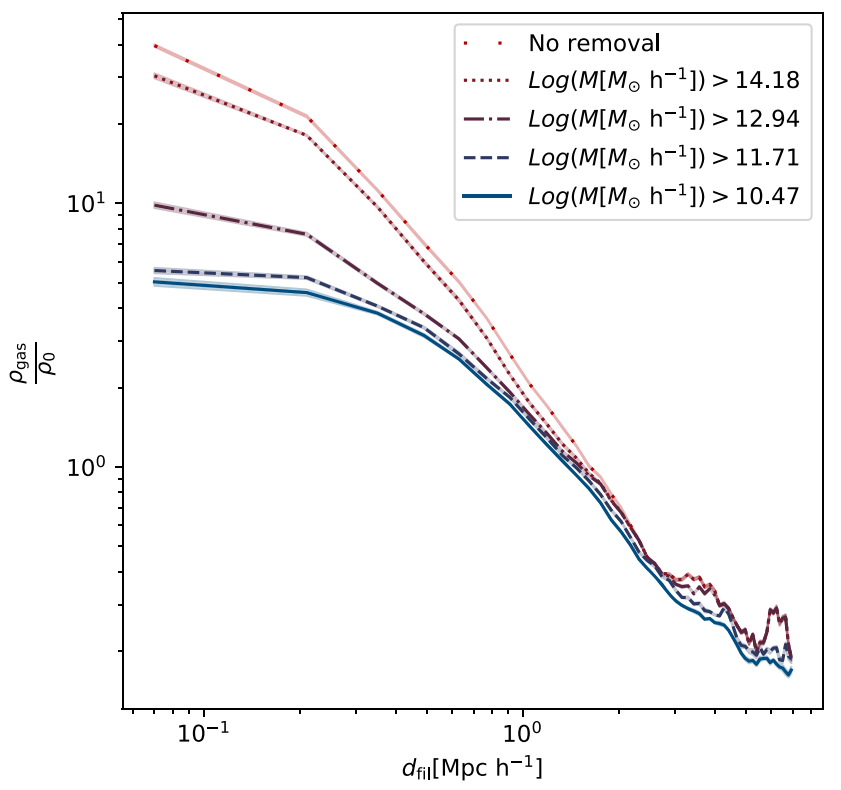

Figure 5. Filament profile and the contribution of haloes: Overdensity profile of gas particles as a function of the radial distance to the nearest filament axis for filaments from the largest cluster. The shape of filament profiles depends on the contribution from haloes, becoming steeper as haloes with progressively higher mass limits are removed. The particles within $2 \times R_{200}$ of haloes with a certain indicated minimum dark matter mass are excluded.

closer to nodes, while the gas density is largely unchanged. Note that the filaments were identified before the removal process.

We also investigate the impact of haloes of different masses. This is highlighted in Fig. 5, which shows the profiles of gas filaments after removing volumes with radii of $2 \times R_{200}$ surrounding progressively more massive haloes as indicated in the caption. We explain the 
procedure of obtaining profiles in the next subsection. The largest halo in our volume has a mass of $10^{15.41} h^{-1} \mathrm{M}_{\odot}$, representing the main cluster itself. Massive haloes constitute the majority of the filament density (top lines in Fig. 5). This levels off at lower masses. Since removing haloes above a mass of $10^{10.47} h^{-1} \mathrm{M}_{\odot}$ and above a mass of $10^{11.71} h^{-1} \mathrm{M}_{\odot}$ (the two lower profiles) does not substantially modify the filament-perpendicular profile, we decide to remove all haloes with masses above $10^{11} h^{-1} \mathrm{M}_{\odot}$ for the removal process. In this process, due the resolution limit of our simulations, we know that we will work with environments that may lack of physics that is not fully resolved (i.e. turbulence). However, as described in the next sections, we calculate general properties (by averaging or stacking) that will erase the small-scale fluctuation making our results robust.

In summary, we conclude that removing volumes equivalent to $2 \times$ $R_{200}$ around haloes with halo masses larger than $10^{11} h^{-1} \mathrm{M}_{\odot}$ largely uncovers the signal of the filaments themselves, and it is this value that we use for the remainder of this paper. While we continue our analysis by reviewing filament material after removing particles in these regions, we acknowledge that this is a choice that comes with drawbacks. We expect some degree of gas cooling and increased density due to shocks of the spine of filaments (Dekel et al. 2009) that in turn helps to build-up haloes and galaxies more efficiently near the spine. This leads to a correlation between halo and filament positions that adds a challenge to distinguishing between pure spine flows and halo outskirt particles. These cooler flows are an integral part of the filament and by excising massive haloes, we inevitably also remove some filament gas along the spine.

\subsection{Filament density profile determination}

We first define important quantities (see Fig. 6) needed for the determination of filament profiles. One such quantity is $d_{\text {fil }}$, the perpendicular distance of every gas and dark matter particle to the nearest filament segment. ${ }^{4}$ Note that particles whose closest segment is the final segment (filament end) are dropped from the analysis as these particles do not have a perpendicular distance to the filament itself, lying beyond the end of the structure. This approach is in line with other studies, including work by Kraljic et al. (2018), Malavasi et al. (2017), and Tanimura et al. (2020). It has the advantage of automatically following curved filaments over the alternative approach of imposing a cylindrical region of interest (adopted by, e.g. Colberg, Krughoff \& Connolly 2005; Martínez, Muriel \& Coenda 2016; Tanimura et al. 2019). In addition, this means that only the nearest filament to each particle is considered, avoiding signal contamination from other filaments.

In order to statistically study the influence of nodes on gas and dark matter in filaments, we stack the signal across all simulations. To do so, we need to (1) characterize node properties and (2) calculate and normalize the distance of any given particle to the node along the spine of the filament (see Fig. 6). Characteristic quantities like node size or mass are not identified by the DISPERSE algorithm. As an approximation, we find all haloes within a sphere of radius $0.4 h^{-1} \mathrm{Mpc}$ centred on each of the node's position as retrievd by DISPERSE. The properties of the most massive halo in this area act as a proxy for the node. In this way we can separate nodes into small, medium and large nodes in the following sections. We further define $d_{\text {node }}$ as the distance along the filament of a particle's closest segment to the node (Fig. 6), normalized by $R_{200}$ of the halo node. Note that

${ }^{4}$ Filaments are described by DISPERSE as a skeleton made up of a succession of short segments.

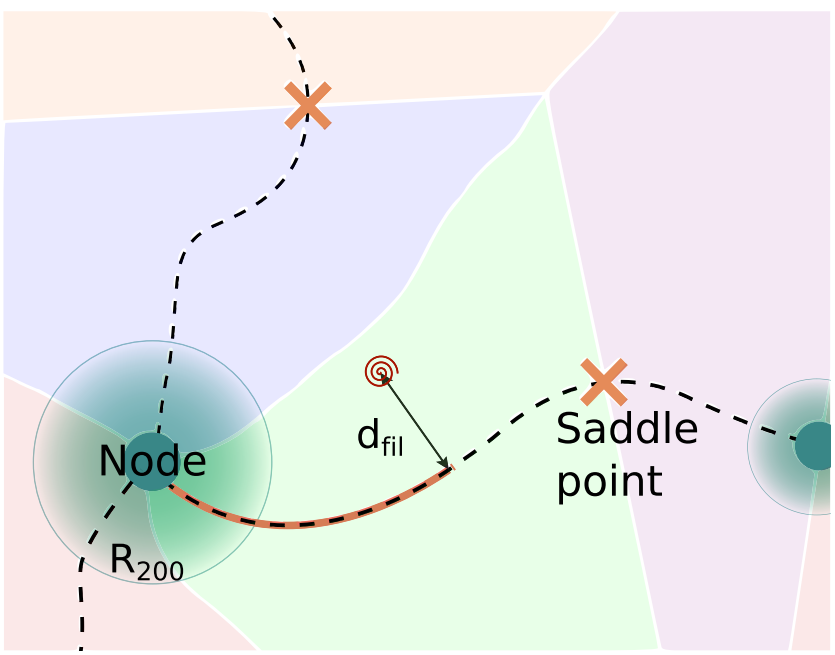

Figure 6. Diagram of the quantities used in this work: Illustration of a section of a network displaying nodes with their $R_{200}$ spherical region, saddle points - marked with ' $x$ ' - and the filaments as dashed lines. An object's $d_{\text {fil }}$ is defined as the perpendicular distance to the closest filament, whereas $d_{\text {node }}$ is defined as the distance to the node along the filament (indicated by the orange line). We present these values normalized by $R_{200}$.

$d_{\text {node }}$ does not allow us to easily study saddle points, as in terms of $d_{\text {node }}$, the positions of the saddle structure will vary from each filament.

The perpendicular filament density profiles in this paper are presented separately for gas and dark matter particles. We calculate them by dividing the count of particles in bins of distance to the filament $\left[N_{\mathrm{p}}\left(r_{\mathrm{i}}, r_{\mathrm{i}+1}\right)\right.$, for $\mathrm{p}$ particles], and stacking over numerous populations of filaments. We then normalize by the total volume occupied by the particles in each bin. This is estimated by using a set of $N_{0}=5000000$ randomly positioned particles occupying the same spherical volume of radius $10 \mathrm{Mpch}^{-1}$ and determining the number count in the same distance bins $N_{\text {random }}\left(r_{\mathrm{i}}, r_{\mathrm{i}+1}\right)$.

Then, the overdensity profile of each type of particle $\mathrm{p}$ can be defined as

$\rho_{\mathrm{p}} / \rho_{0}=\frac{N_{\mathrm{p}}\left(r_{\mathrm{i}}, r_{\mathrm{i}+1}\right)}{N_{\text {random }}\left(r_{\mathrm{i}}, r_{\mathrm{i}+1}\right)} \frac{N_{0} m_{\mathrm{p}}}{V_{0} \rho_{0}}$,

where $N_{0}$ is the amount of random particles in the total volume $V_{0}$, $\rho_{0}$ is the average cosmic density, and $m_{\mathrm{p}}$ is the mass of the particle. When applied to all the particles in the full volume this formula gives us the overdensity of the filament, but it includes a high degree of contamination from the enclosed haloes.

The multiplicity of filaments, their faint signal compared to highdensity structures, and the added difficulty as a result of the removal of haloes (Section 3) challenges the study of individual filaments. We thus infer the statistical properties of populations of filament (e.g. a set of long filaments, or all filaments connected to the centre of a cluster) by employing the stacking method. With this, all filaments in the sample contribute to the signal that we measure, thereby improving the signal-to-noise ratio and removing small-scale fluctuations that we may not be well resolving by lack of resolution.

For results presented in the following sections, we have therefore combined the particles of all 324 cluster realisations, and separated by different populations of filaments (e.g. short and long filaments, as explained in the following). The high number of filaments (11 058) ensures meaningful statistical samples. We estimate the standard deviations of the density/fraction curves with the Jackknife method. We build 324 samples of 323 clusters each, excluding the $i$ th cluster, 


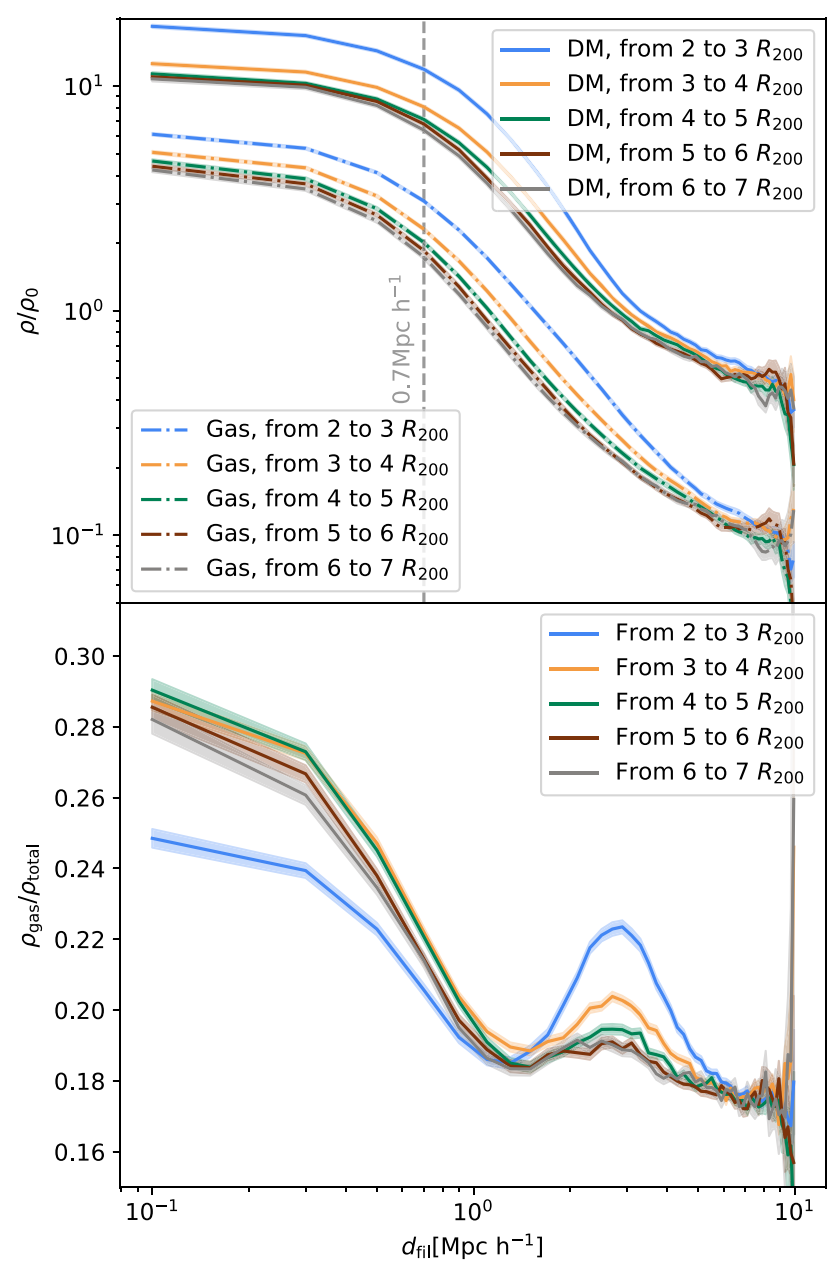

Figure 7. Filaments are thicker closer to nodes. Top: Stack of density profiles perpendicular to the filaments for gas and dark matter particles normalized by the cosmic average density $\rho_{0}$. The solid lines indicate the dark matter profile, and the dashed lines the gas profiles. Various colours differentiate distance from the filament node. Bottom: gas fraction, determined as the ratio between the gas profile and the total DM + gas profiles. Only filaments with nodes and saddle points and without bifurcation or exit points were stacked. Particles within $2 \times R_{200}$ of haloes above a minimum mass of $10^{11} h^{-1} \mathrm{M}_{\odot}$ are not considered (see text for details). The estimated standard deviation of the curves is shown as a shade of the same curve's colour.

and recalculate the profiles/fractions. In contrast with rather building samples of filaments, with this method we allow the cosmic variance to contribute to the error of the signal, since the total cluster masses differ significantly between each other.

\subsection{Dark matter and gas characteristics of cluster filaments}

The density profiles allows us to quantify a characteristic filament 'thickness' at local haloe-scales. However, filaments are not uniform and their thickness may vary depending on proximity to nodes and depend on the used mass proxy, either gas or dark matter. In the following section, we investigate these variations. In our scenario, nodes typically mark the positions of large haloes akin to galaxy clusters and groups, towards which matter is flowing via intercluster filaments. In Fig. 7, we split particles (both dark matter and gas) into six bins of $d_{\text {node }}$ : [2, 3], [3, 4], [4, 5], [5, 6], and [6, 7], indicated by lines of different colours. The dot-dashed lines show profiles for gas particles and solid lines for dark matter particles.

The top panel in Fig. 7 reveals a 'cored' radial density profile up to approximately $0.7 h^{-1} \mathrm{Mpc}$ distance to the filament axis, as marked in the figure. We use this value as a 'typical thickness' for clusterfilaments as revealed by THE THREEHUNDRED. At larger distances, the profiles follow a power law $r^{-\gamma}$, with $\gamma \approx 2$. A similar shape is also seen in other works with different emphases and starting points, e.g. Colberg et al. (2005) who used $\triangle C D M$ numerical simulations to study the filaments between pairs of clusters, and Dolag et al. (2006) who studied gas density profiles in hydrodynamical resimulations (see also Aragón-Calvo, van de Weygaert \& Jones 2010; also Bonjean et al. 2019; Galárraga-Espinosa et al. 2020). Note, however, that the shape of the gas density profile is resolution and cooling model dependent.

It is not trivial to define a typical 'thickness' of filaments, let alone compare between different studies, since this depends on individual definitions. In the literature, some studies determine the radius of a filament as the distance from which the density profile starts to follow a power law (e.g. Colberg et al. 2005; Aragón-Calvo et al. 2010). They find a value for a characteristic filament radius of $\approx 2 h^{-1} \mathrm{Mpc}$. Others define the distance at which the density drops below a certain threshold (Dolag et al. 2006), which leads to higher values of $5 h^{-1} \mathrm{Mpc}$ radius. Yet others use the scale parameters that fit the data using a certain model, finding thickness parameter values of $r_{2}=$ $2-3 h^{-1} \mathrm{Mpc}$, with long filaments being thinner than short filaments (Galárraga-Espinosa et al. 2020), or $r_{m} \approx 7.5 \mathrm{Mpc}$ for a exponential decay fit for long filaments $>20 \mathrm{Mpc}$ (Bonjean et al. 2019).

For gas and dark matter alike, the peak overdensity rises towards the nodes by a factor of around 2 (indicated by different coloured lines). This could be due to the remaining influence of the central cluster that extends beyond the radius we use to remove its contribution $\left(2 \times R_{200}\right)$. The thicker filaments closer to nodes could therefore be related to halo outskirt particles that mix with filament particles, or rather be a genuine correlation of the filamentary structure and the proximity to a node. This effect disappears beyond three times $R_{200}$. This observations is also seen in other studies, e.g. Dolag et al. (2006), who also find that gas profiles increase to higher values closer to nodes. Furthermore, the power law $r^{-\gamma}$ that profiles follow changes from $\gamma \approx 3$ near the node to $\gamma \approx 2$ further away. This is consistent with a divergence from an NFW profile at the node Navarro, Frenk \& White (1996). The varying peak density and width are also seen in Kraljic et al. (2019), with higher densities towards the nodes in the galaxy distribution and lower width of the isocontours at the saddle point.

Fig. 7 also shows that local properties of the filament profiles from gas particles (the dot-dashed lines) differ from those based on dark matter particles (the solid lines), where dark matter filaments are somewhat thicker, i.e. they appear less concentrated than gas filaments. This is especially noticeable for bins $[2,3]$ and $[3,4] R_{200}$ distance from the node, where the drop is noticeably steeper for dark matter particles. This could imply that the gas around haloes is more homogeneous than dark matter or that dark matter particles depend more strongly on the distance to the node. Alternatively, gas filaments around haloes could be more concentrated due to the ability of the gas to shock and cool while dark matter is collisionless (see also Fig. 3 in which the dark matter filaments appear 'fluffier'). This can have important implications for the galaxies themselves (Birnboim \& Dekel 2003; Pichon et al. 2011; Danovich et al. 2012) and their distribution in clusters (Welker et al. 2020). Note that this result refers to massive clusters at $\mathrm{z}=0$, where gas filaments could be more perturbed than at higher redshifts (Dubois et al. 2012). This difference 


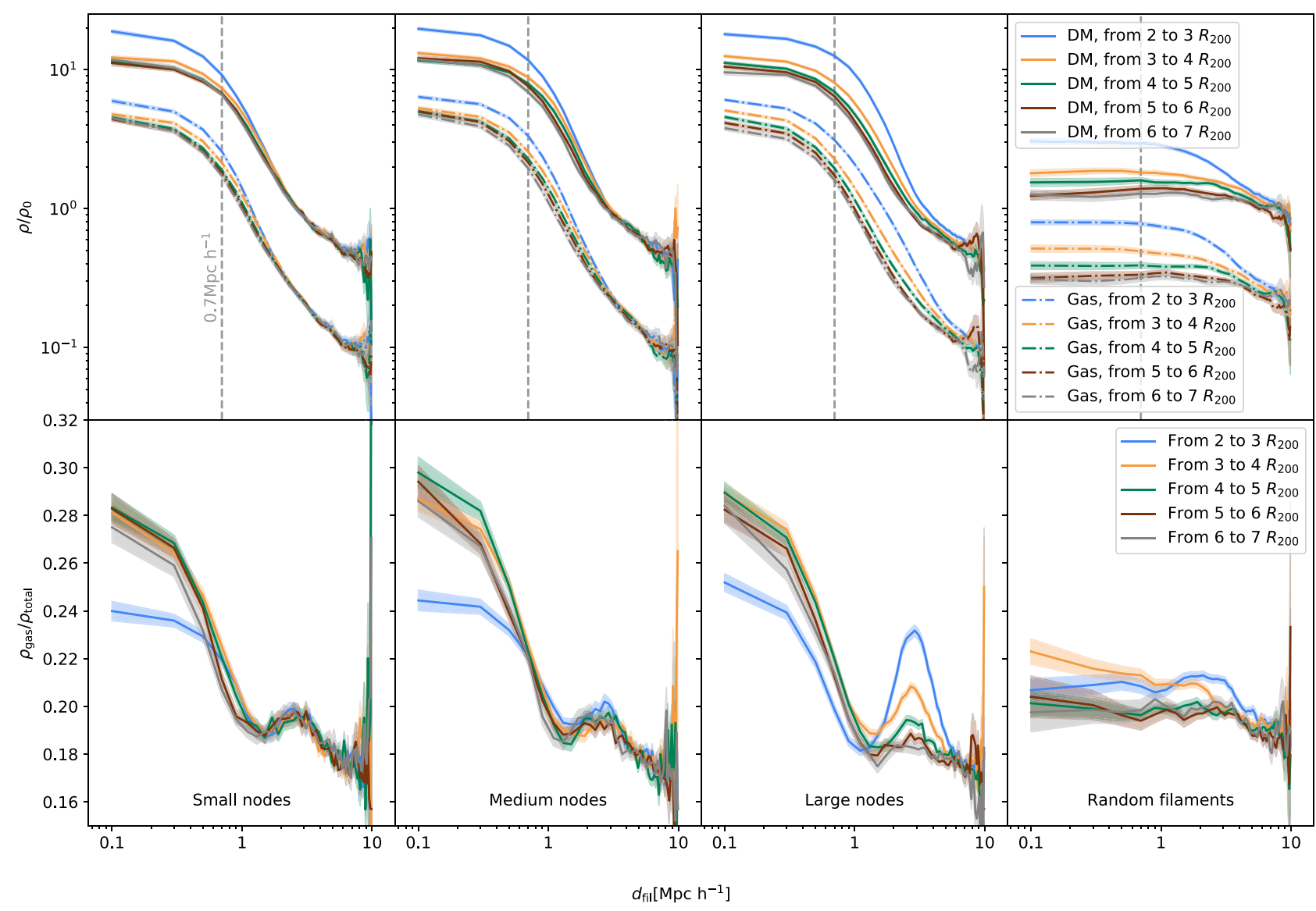

Figure 8. Combined effect of haloes and distance to nodes: Perpendicular density profiles for stacked filaments. As for 7 but split by node size. Left column displays the smallest nodes while the third column displays the largest nodes, usually the central haloes of each simulation. The last column shows the density profiles of filaments that had been randomly rotated about the node (see text for details).

between gas and dark matter is more pronounced in profiles of gas fractions $\left(\right.$ fraction $=\frac{\rho_{\text {gas }}}{\rho_{\text {gas }}+\rho_{\mathrm{DM}}}$ ), which we show in the bottom panel of Fig. 7: while the gas fraction rises towards filaments as expected, a notable bump at a distance of around 2-3 Mpc from the filament is evidently appears. Its peak fraction varies with the distance to the node, indicated by the coloured lines. This increased gas fraction closer to the cluster (the 'bump') seems to be related to a turbulent interaction generated by the convergence of gas falling into the cluster through filaments and gas falling in isotropically. In a later section (Section 4.1), we argue that this feature originates from the different dynamical signatures of gas and dark matter, as will become evident through their velocity fields. Without experiencing pressure and contractions from cooling, dark matter is able to reach higher velocities. Note, however, that the increase of gas fractions towards filaments seen here is not found in a study of filaments at larger scales and less dense environments by Gheller \& Vazza (2019). In their study, the authors found that the baryon fraction enclosed in filaments normalized by the cosmic mean depends with the filament mass, barely varies with different feedback models and tends to be below the unity at around 0.9 . One reason for this discrepancy could be that we base our filament positions on gas particles. However, differences also could be due to cluster outskirt physics: the dense environments, turbulent mixing of material and thick, perturbed filaments feeding the clusters marking this an unexplored territory.

\subsection{The combined effect of nodes and distance along filaments}

Distance to filaments and distance to node are not independent measures. Gas and dark matter densities drop with increasing distance from the nodes (roughly speaking, with distances along the filament spine). Densities therefore drop with orthogonal distances to the filament axes as well as with distances from the node. In addition, densities - and therefore filament profiles - might change with the prominence (mass and size) of the node (e.g. groups and clusters). In order to investigate this interdependence, we split the filament sample into three roughly equal groups according to the size of the node they are connected to. This is comparable to splitting by halo mass. The three ranges of $R_{200}$ were $(0.0,0.45) h^{-1} \mathrm{Mpc}$ for the smallest, $(0.45$, $0.61) h^{-1} \mathrm{Mpc}$ for medium sized, and $(0.61,2.25) h^{-1} \mathrm{Mpc}$ for the largest nodes. The last bin includes all central haloes, i.e. the massive cluster at the centre of the simulated regions. Density profiles and gas fractions for different node sizes are shown in the first three columns of Fig. 8 and lines and distance to node bins are the same as in Fig. 7. As we go to large-sized nodes, filaments vary more at different radii (distances to the nodes), the typical standard deviation between the profiles up to $d_{\text {fil }}=1 \mathrm{Mpc} \mathrm{h}^{-1}$ is $\approx 0.4, \approx 0.5$, and $\approx 0.63$ for the gas for Small, Medium, Large nodes and $\approx 1.67, \approx 2.13, \approx 2.56$ for the dark matter case, respectively. This means that filaments around clusters are thicker closer to the cluster whereas filaments around smaller nodes show very little variation of thickness. 
As mentioned above, the $d_{\text {node }}$ and the distance to the centre of the cluster are not independent of one another. Our goal is to disentangle the contribution of the density profile from the massive node at the centre from the density profile from filaments. For this, we rotate the true filament networks using random angles and re-calculate the density profiles of these random filaments. Results are shown in the final column of Fig. 8, labelled 'random filaments'. The profiles are flat closer to these random filaments and drop off at about $\sim 3 h^{-1} \mathrm{Mpc}$ away from them. This suggests that up to $d_{\text {fil }} \sim 3 h^{-1} \mathrm{Mpc}$, gas is influenced by the filaments themselves. At larger distances from the filaments, density profiles are driven by the clusters (central nodes). The distance to the filament, however, plays a far more important role than the distance to the cluster, which only features as a minor component in the density profiles.

The lower panel in Fig. 8 shows gas fractions as before and reveals that the 'bump' (increased gas fractions closer to nodes) at around $2-3 h^{-1} \mathrm{Mpc}$ away from the filament spine we described in the previous section is most prominent close to large, cluster-sized nodes. This is the region around clusters where filament contribution and cluster contribution compete. Further away from the cluster, the 'bump' is suppressed. Around smaller nodes (e.g. groups or larger galaxies), this 'bump' is much more restrained and constant at all distances from the node. We will explore this feature in more detail in the next section.

\section{THE VELOCITY WEB AROUND GALAXY CLUSTERS}

The complex cold gas dynamics of the large-scale structure is governed by the successive formation of walls, filaments, and clusters (Zel'dovich 1970; Bond et al. 1996; Pichon et al. 2011). In this framework, matter is falling towards a filament from the wall in which the structure is embedded. As a result of these large-scale flows, vortices can form in a specific area perpendicular to the filament axis and in the direction of the wall. Simultaneously, matter flows away from saddle points and towards nodes. This is the complex environment in which galaxies form and evolve. As a consequence, galaxy properties such as orientation of the spin and disc alignments are affected by the winding of the walls into filaments in particular ways (Aragón-Calvo et al. 2007; Sousbie, Colombi \& Pichon 2009; Codis et al. 2012; Libeskind et al. 2012).

In our study of the velocity field around filaments in THE THREEHUNDRED, we first account for the presence of haloes and other virialized structures (see discussion in Section 3.2 for the removal process). The removal of particles related to haloes allows us to investigate the motion of the diffuse gas and dark matter towards filaments and - tangential to it - towards nodes. The filaments we study converge on to clusters, which leads to a radial pattern of the network. As a consequence, material that is moving towards filaments will simultaneously also move radially towards the cluster. In order to isolate velocities, we first need to control for this systematic bias.

\subsection{Accretion on to clusters: the contribution of filaments}

In order to disentangle the contribution of the filaments from that of the cluster, we first remove the radial component of the infall to the cluster with the following procedure: we transform the velocities to the galaxy cluster's frame of reference. Assuming that the infall velocity depends on the distance to the cluster, we determine the average infall profile in 50 bins in the range $[0,10] h^{-1} \mathrm{Mpc}$. This radial velocity field is then used to adjust the velocity of each particle

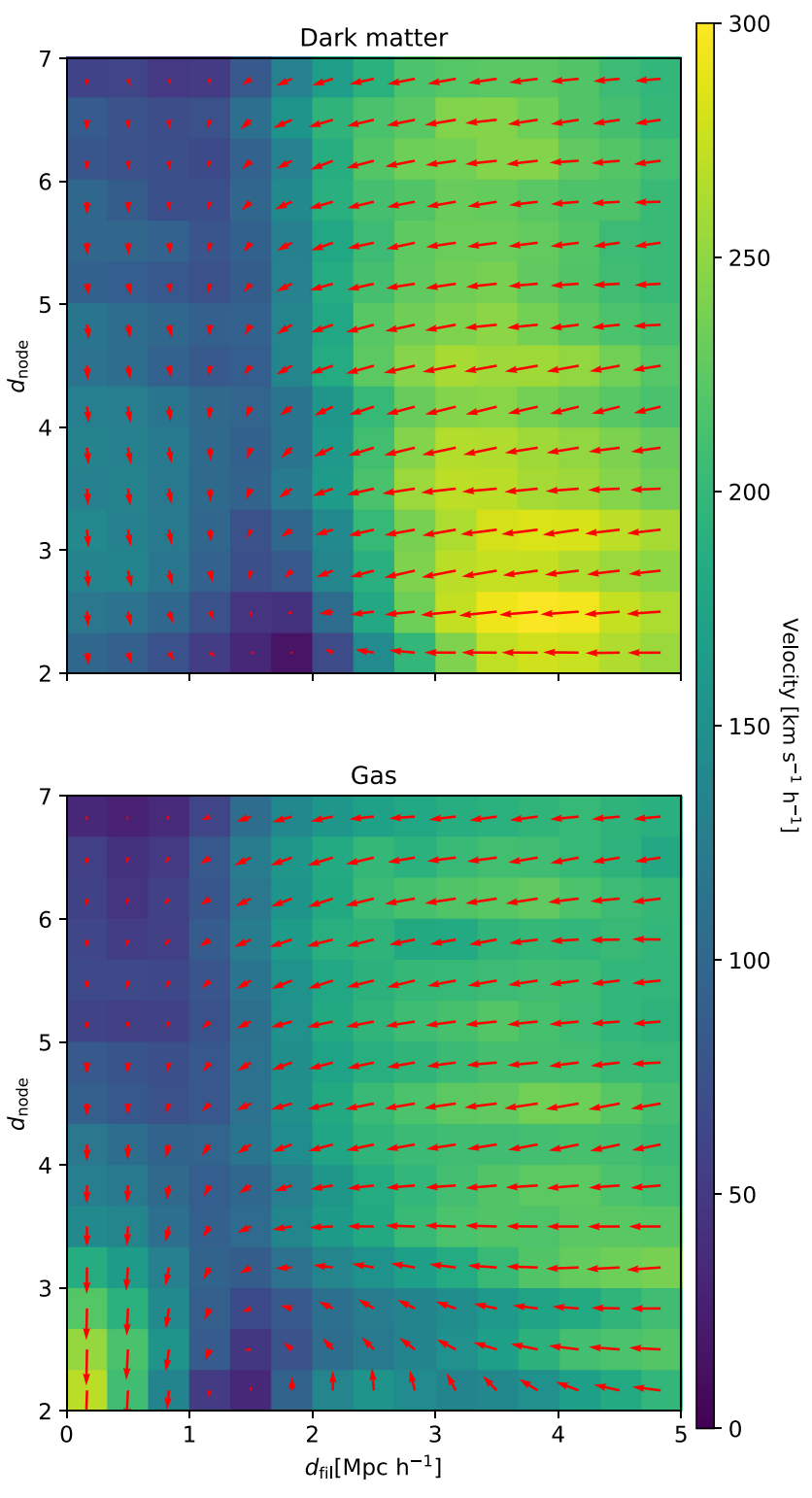

Figure 9. Dynamics of filaments: Velocity field of dark matter (on the top) and gas (on the bottom) around filaments. Particles within twice $R_{200}$ of haloes more massive than $10^{11} h^{-1} \mathrm{M}_{\odot}$ have been excised and the radial flow into clusters has been subtracted (see text for details).

according to its distance from the cluster centre. We do this for gas and dark matter separately.

After removing the cluster's imprint on the global velocity field, we identify the nearest filament segment to each particle and calculate the radial velocity component (defined by the vector from the closest segment to the particle), and tangential velocity component (defined by the direction of the closest filament segment axis, towards the node). As before, we only include complete filaments with both a node and a saddle point and without bifurcation or exit points in this analysis.

Fig. 9 shows the stacked result of the velocity field of dark matter (top) and gas (bottom) in all 324 cluster volumes of the sample. It shows the radial and tangential velocity components in bins of distance along the filament spine and in bins of distance to the filament axis. The colour coding indicates the velocity in $\mathrm{km} \mathrm{s}^{-1} \mathrm{~h}^{-1}$. Immediately, we can see a smooth collective motion of matter from lowdensity regions towards filaments and nodes. Furthest away from fil- 
aments and nodes, the velocity fields of dark matter and gas look similar. However, near the nodes the motion of gas deviates from that of the dark matter: close to the intersection of filaments and nodes, a vortex of gas forms - which is absent in collisionless dark matter. This is consistent with the observation that gas resists the shock in filaments.

We further investigate this feature in Figs 10 (i) and (ii), where we fold in information of gas fractions (shown by the colour coding) and plot isocontours of the tangential component of the velocity (i.e. towards the nodes and parallel to the filament). Fig. 10 (i) details the velocity flows of gas and dark matter for different filament lengths and 10 (ii) shows different node sizes separately. In this way, we link gas motions to the increase of gas fractions that we first saw in the radial profiles in Figs 7 and 8. The negative tangential velocity component close to nodes and near filaments in all plots exposes the parallel to the filament motion towards nodes. However, only in the plots of the gas we see a region of positive velocities close to nodes and filaments that coincides with higher gas fractions. This is consistent with our findings in Figs 7 and 9 and is absent in dark matter. The positive velocity region (and hence the gas fraction bump) is seen for all lengths, and it is stronger for the longest filaments bin (Fig. 10i) and for the large nodes (Fig. 10ii). Given the dependence of filament lengths and node size (Fig. 2), this underlines the observation that this phenomenon is related to the central nodes.

\subsection{Accretion on to filaments}

In the previous section, we detailed the velocity field in cluster outskirts that is driven by both an infall towards the nodes and a collapse of filaments. In order to isolate the motion towards filaments, we now consider the orthogonal distance to the filament axis and the radial velocity component of gas and dark matter particles. This is shown in Fig. 11, where we plot phase-space diagrams for gas on the left and dark matter on the right-hand hand side. Both show a similar behaviour: a collective movement of particles falling towards the filament at $\approx 250 h^{-1} \mathrm{~km} \mathrm{~s}^{-1}$ (indicated by negative velocities) from larger distances, and large random motions inside the filament (as indicated by the vertical dashed lines). This plot is quite similar to the ones done stacking haloes (Oman, Hudson \& Behroozi 2013; Arthur et al. 2019; Hamabata, Oguri \& Nishimichi 2019), but in this case we integrate along the filament axis and stack afterwards. In this way, we see how this structures that as a whole are not yet virialized, when we integrate their properties in a symmetric direction, we recover virial properties. However, this apparent virialized region is more likely to be linked to the motion of material that is shell crossing for the first time, or related to material formerly bounded to haloes. For dark matter, we see a broader but still symmetric range of velocities at the core compared to the gas, but a slight increase in dispersion near the centre. Fig. 7 already illustrated the 'fluffier' character of dark matter filaments compared to gas filaments. This is consistent with the ability of dark matter to cross-shell material when it is being accreted to the spine while the gas shocks and cools down.

Taking this further we split our filament sample into the same three groups as used before, depending on node size. Although not shown in this paper, as we move to progressively larger nodes, we observed an increase in the width of the velocity range, as expected for the mass enclosed in those filaments.

We tested our procedure by repeating the analysis after first randomly rotating the filamentary networks about each central node. As expected, this completely erases the correlation with the filament's distance, and the radial infall on to the filaments vanishes.

\subsection{Filaments as gas highways}

Finally, we investigate how dark matter and gas move inside and outside filaments as a function of distance to the cluster centre. For this, we only consider galaxy clusters that are dynamically relaxed (as defined by Haggar et al. 2020), since we consider that the anisotropic contribution of a perturbed halo will affect our comparison of an isotropic smooth accretion and the one through filaments. The relaxation parameter is calculated using a combination of the virial ratio, the centre-of-mass offset and the fraction of cluster mass in subhaloes (see also Cui et al. 2018b, for details), in this section we include clusters with a relaxation parameter greater than one.

In Fig. 12, we separate dark matter and gas particles each into two groups: particles inside filaments have distances to skeletons smaller than $1 h^{-1} \mathrm{Mpc}$ (the blue solid line), and particles with larger distances are defined as outside filaments (the red dot-dashed line). To obtain the curves, we calculated radial velocities for all particles and stacked results of all relaxed clusters. From top to bottom, we show radial velocity components in three shells of increasing distance from the cluster centre. The inner and outer radii of these shells are [2, 2.66], [2.66, 3.33], and [3.33, 4]. As before, all particles within $2 x R_{200}$ of haloes are excluded from this analysis.

Furthest away from the cluster (bottom left-hand panel in Fig 12), dark matter, and gas move towards nodes at a rate that is largely independent of whether or not a particle is inside or outside a filament, the medians of the distributions, marked as the vertical lines of the same colours, indicate velocities around $900 h^{-1} \mathrm{~km} \mathrm{~s}^{-1}$. Here, only a small fraction of particles (and more so in gas than in dark matter) has velocities close to $0 h^{-1} \mathrm{~km} \mathrm{~s}^{-1}$ or positive velocities, indicating outflows. This changes as we go closer to the cluster: the middle right-hand panel reveals that even at larger distances from the cluster, gas is partially stationary (i.e. this component does not move with respect to the cluster), something not seen in dark matter. Just outside $2 \times R_{200}$ of the node, a clear dichotomy emerges. Both dark matter and gas are split between particles that are moving into the cluster and particles that have a symmetric velocity distribution centred on $0 \mathrm{~km} \mathrm{~s}^{-1}$, which could correspond to an accretion shock just outside the cluster. Furthermore, we see increasing differences between gas and dark matter (differences of the median of $\approx 780 h^{-1} \mathrm{~km} \mathrm{~s}^{-1}$ for the gas, meanwhile for the dark matter it is $\approx 280 h^{-1} \mathrm{~km} \mathrm{~s}^{-1}$ ). Gas is falling into nodes with a clear preference to be inside filaments (negative velocities, blue curve), but leaves the cluster with a clear preference of being outside filaments (positive velocities, the red curve). For dark matter, this preference is far less obvious. This observation is consistent with Figs 10 (i) and (ii), where we saw a region of negative tangential velocities near the filamentary axis and a region of positive velocities outside the filament.

The behaviour we see in Fig. 12, could be due to random motions of particles in clusters on the one hand, but also to an increasing presence of accretion shock and/or backsplash material close to clusters (e.g. Haggar et al. 2020). The latter refers to material that has gone through the cluster and will return on a second infall. This plot confirms that filaments act as highways to funnel material into clusters (Cautun et al. 2014; Kraljic et al. 2018), however, with noteable differences between dark matter and gas. Gas seems to penetrate the expanding shock shell of clusters more efficiently when it is flowing through a filament, while the diffuse dark matter falling into clusters through filaments does not differ from an isotropic infall. Gas in filaments seems to be less sensitive to shocks, a phenomenon that once motivated the study of cold flows on smaller halo scales, but 


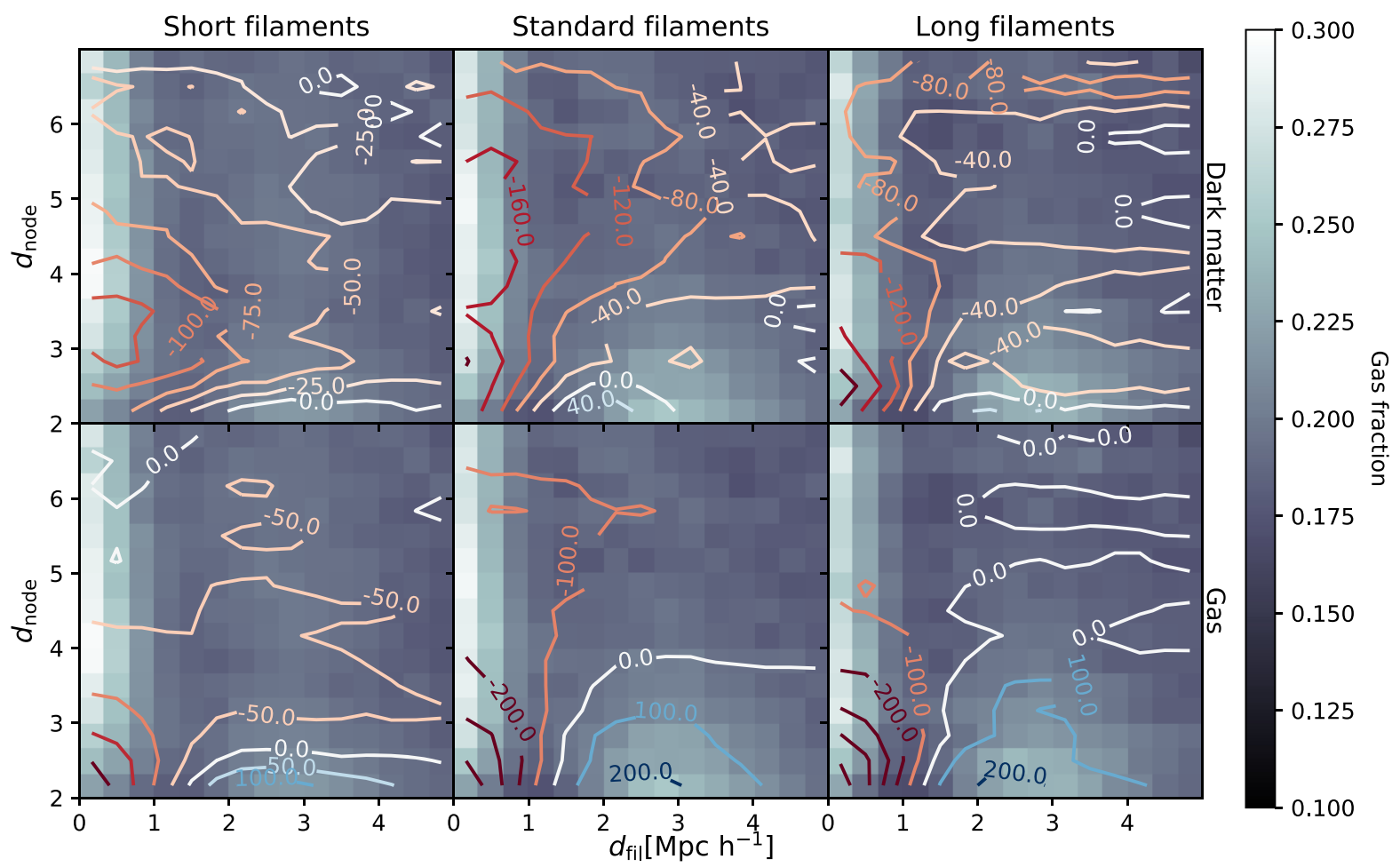

(i) Tangential component of velocity along the filamentary axis separated by different filaments lengths

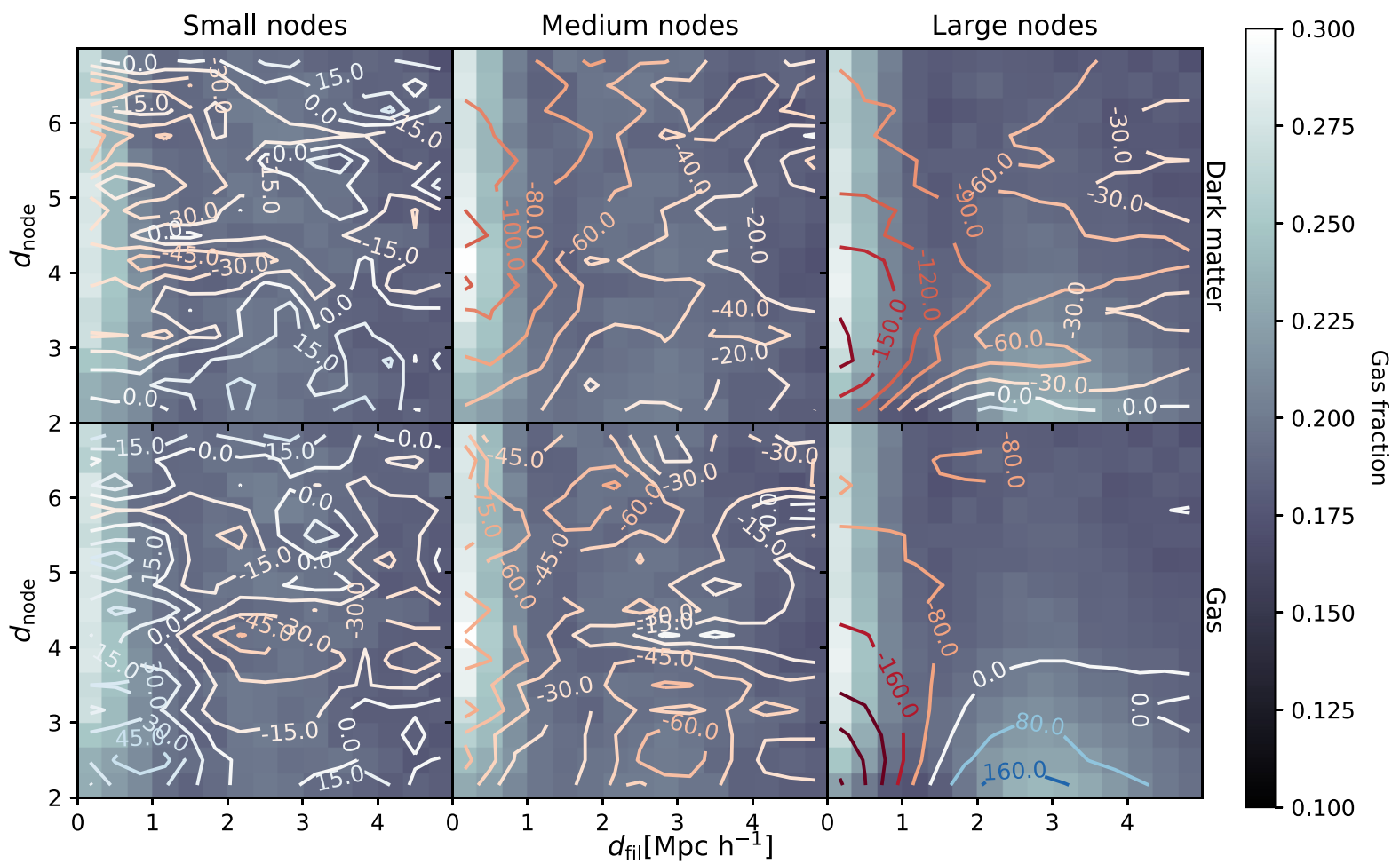

(ii) Tangential component of velocity along the filamentary axis separated by different node sizes

Figure 10. Dynamics of gas and dark matter near the centre of the cluster. Top: Tangential component of velocity along the filamentary axis separated by different filaments lengths: short, standard, long. The $x$-axis indicates distance to filament in $\mathrm{h}^{-1} \mathrm{Mpc}$, the $y$-axis indicates $d_{\text {node }}$ short: Lengths between 2-5 $\mathrm{h}^{-1} \mathrm{Mpc}$; standard: 5-8 $\mathrm{h}^{-1} \mathrm{Mpc}$; long: $8-14 \mathrm{~h}^{-1} \mathrm{Mpc}$. Bottom: Tangential component of velocity along the filamentary axis separated by different node sizes: small, medium, big. The $x$-axis indicates distance to filament in $\mathrm{h}^{-1} \mathrm{Mpc}$, the $y$-axis indicates $d_{\text {node }}$. Small: Node's $R_{200}$ is between $0-0.45 \mathrm{~h}^{-1} \mathrm{Mpc}$; medium: $0.45-0.61 \mathrm{~h}^{-1} \mathrm{Mpc}$; large: $0.61-2.3 \mathrm{~h}^{-1} \mathrm{Mpc}$. 


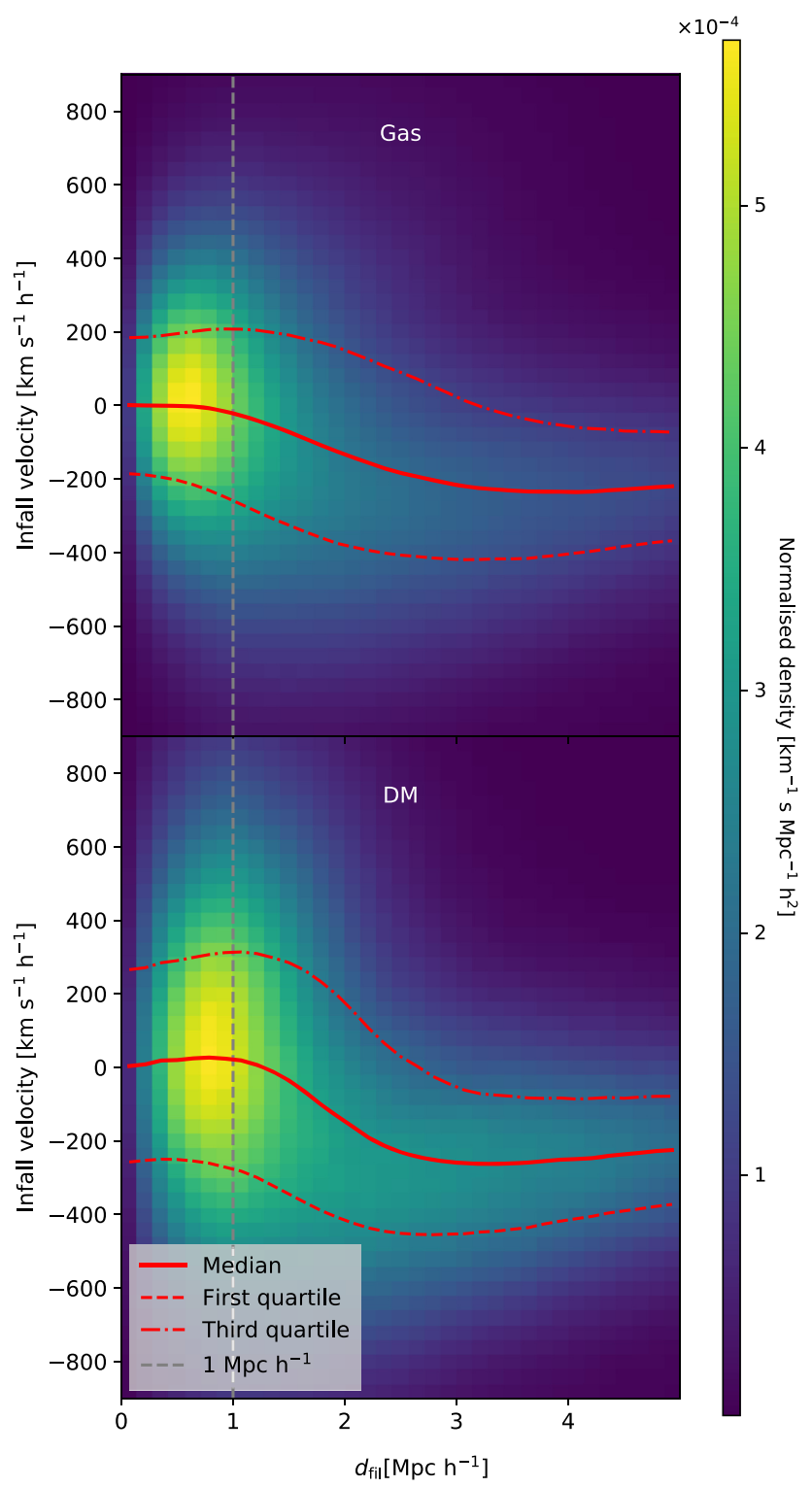

Figure 11. Accretion on to filaments: Phase position-velocity diagram for dark matter and gas particles around filaments after removing cluster infall. The $x$-axis is the distance to the filament axis and the $y$-axis is the velocity radial component. The red lines indicate the median and the first and third quartiles of the distribution. The vertical grey-dashed line indicates a filament thickness of $1 h^{-1} \mathrm{Mpc}$. The colour represents the value of the distribution function.

also theoretical studies up to cluster scales (Cornuault et al. 2018). Moreover, the different behaviour of gas and dark matter is most prominent close to clusters, and becomes less evident further away.

\section{CONCLUSIONS}

In this paper, we have analysed filaments detected by DISPERSE in the environments around a set of 324 cluster re-simulations of THE THREEHUNDRED project. We determined the key characteristics of these structures such as the gas and dark matter profiles and velocity flows. We clean the filaments excluding all material inside $2 \times R_{200}$ radius of all haloes with masses above $10^{11} h^{-1} \mathrm{M}_{\odot}$. In this way, we aim to study unbound material (either gas or dark matter) that

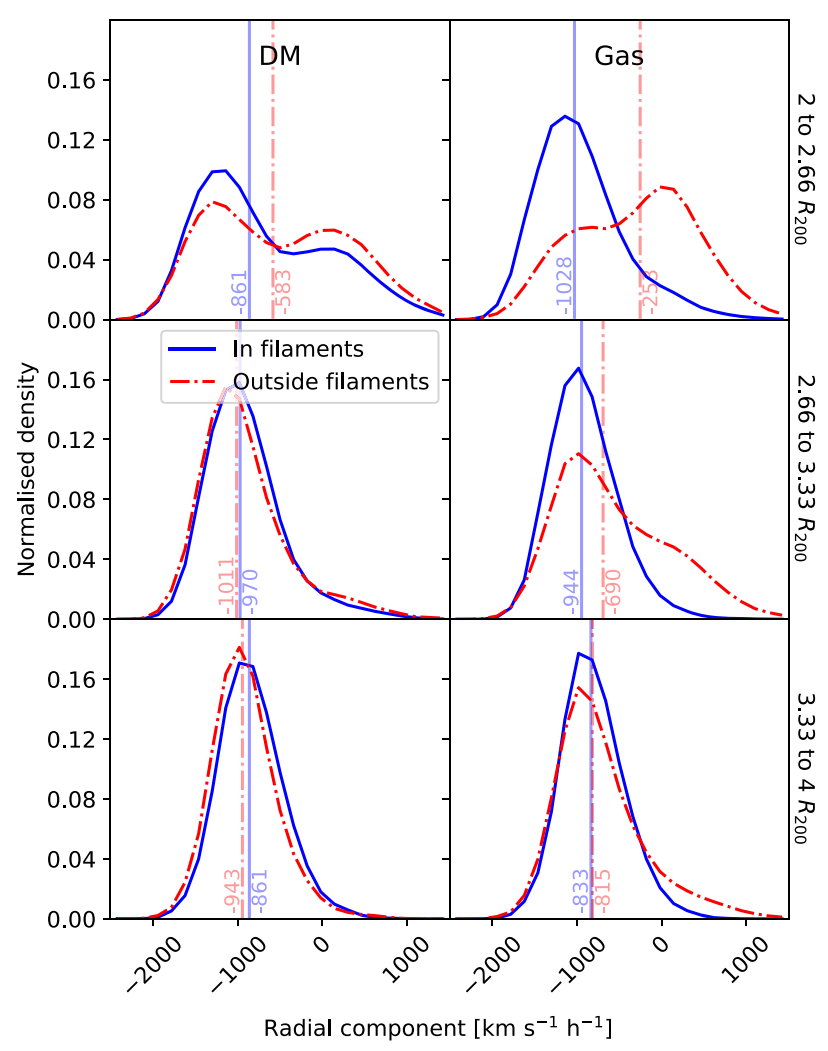

Figure 12. Filaments as gas highways: Normalized histogram of the radial infall velocity to the cluster. From top to bottom: particles in the ranges [2, 2.66], [2.66, 3.33], [3.33, 4] $\times R_{200}$ from the centre of the cluster. On the left: dark matter particles. On the right: gas particles. The lines show particles inside filaments (blue) and outside filaments (red). Their respective median of the distribution is marked with a vertical line of the same colour. Only relaxed clusters were stacked (relaxedness $>1$, see text for description.).

constitutes the diffuse material of filaments. We analyse the filaments by stacking and averaging to describe global properties common to them. We found evidence that supports the idea that filaments are structures with well-defined properties in both dark matter and baryons. Importantly, we identified differences between dark matter and gas velocities close to clusters and just outside filaments.

The main conclusions of our analysis are summarized in the following:

(i) The dark matter and gas density profiles of filaments surrounding clusters have similar shapes and features, such as the presence of a 'core' at the filament axis, a density decay consistent with a power law in the outskirts and a decay of the peak density moving away from the filament node. We report a characteristic filament thickness of around $0.7 h^{-1} \mathrm{Mpc}$.

(ii) The differences between dark matter and gas density profiles become evident in gas fractions: the fraction varies and increases towards the filament spine. Furthermore, a local increase at roughly $2 h^{-1} \mathrm{Mpc}$ distance from the cluster in the gas fraction (a 'bump') suggests turbulent gas that is not present in dark matter.

(iii) Filaments are surrounded by a structured velocity field largely as predicted by theory. This needs to be carefully disentangled from the general velocity inflow surrounding large clusters.

(iv) The velocity-space phase diagram of filaments is analogous to that of the clusters. The matterial infalls at around $-250 h^{-1} \mathrm{~km} \mathrm{~s}^{-1}$ in the outskirts and tends to be centred at zero at the spine. There 
are clear differences between the motion of gas and dark matter particles. The velocity dispersion is present in the whole range, but it is higher for the dark matter, showing an increment towards the spine. Meanwhile, due to gas physics, the velocity dispersion of the gas decreases towards the filament.

(v) Filaments form highways along which material preferentially flows into the clusters. We find that the material that is conducted through the filament has higher infall velocity towards the cluster centre than the material falling in other directions. This is particularly noticeable for the gas, most likely due to the gas pressure, in line with the idea of filaments as channels that allow the infall of fresh material towards the central region.

While higher resolution simulations will be able to better constrain our findings, the results clearly point to different paths of dark matter and gas flows towards the central region of galaxy clusters.

\section{ACKNOWLEDGEMENTS}

This work has been made possible by THE THREEHUNDRED collaboration, ${ }^{6}$ which benefits from financial support of the European Union's Horizon 2020 Research and Innovation programme under the Marie Skłodowska-Curie Actions grant agreement number 734374 , i.e. the LACEGAL project. THE THREEHUNDRED simulations used in this paper have been performed in the MareNostrum Supercomputer at the Barcelona Supercomputing Center because of CPU time granted by the Red Española de Supercomputación. UK acknowledges support from STFC. GY and AK acknowledge financial support from Ministerio de Ciencia, Innovación y Universidades/Fondo Europeo de Desarrollo Regional, under research grant PGC2018-094975-C21. WC acknowledges support from the European Research Council under grant number 670193 (the COSFORM project). FS and AR acknowledge the financial support from Consejo Nacional de Investigaciones Científicas y Técnicas (CONICET, Argentina) by PICT-2016-4174 grant and Secretaría de Ciencia y Tecnología de la Universidad Nacional de Córdoba (SeCyT, UNC, Argentina) by Consolidar-2018-2020 grant.

The authors of this paper contributed in the following ways: AR, FS, FRP, UK, and MEG formed the core team. AR performed the analysis that was largely completed during an EU (LACEGAL) exchange visit to the University of Nottingham. CW provided insightful comments that added to the interpretation of the results and ran DISPERSE. GY undertook the simulations with AK generating the halo catalogues.

\section{DATA AVAILABILITY}

Data available on request to THE THREEHUNDRED collaboration. ${ }^{5}$

\section{REFERENCES}

Aragón-Calvo M. A., Jones B. J. T., van de Weygaert R., van der Hulst J. M., 2007, A\&A, 474, 315

Aragón-Calvo M. A., van de Weygaert R., Jones B. J. T., 2010, MNRAS, 408,2163

Arnold V. I., Shandarin S. F., Zeldovich I. B., 1982, Geophys. Astrophys. Fluid Dyn., 20, 111

Arthur J. et al., 2019, MNRAS, 484, 3968

Beck A. M. et al., 2016, MNRAS, 455, 2110

${ }^{6}$ https://www.the300-project.org

${ }^{5}$ www.the300-project.org
Behroozi P. S., Wechsler R. H., Wu H.-Y., 2013, ApJ, 762, 109

Benitez-Llambay A., 2015, Py-Sphviewer: Py-Sphviewer V1.0.0, http://dx.d oi.org/10.5281/zenodo.21703

Birnboim Y., Dekel A., 2003, MNRAS, 345, 349

Bonamente M., Nevalainen J., Tilton E., Liivamägi J., Tempel E., Heinämäki P., Fang T., 2016, MNRAS, 457, 4236

Bond J. R., Kofman L., Pogosyan D., 1996, Nature, 380, 603

Bond N. A., Strauss M. A., Cen R., 2010, MNRAS, 409, 156

Bonjean V., Aghanim N., Douspis M., Malavasi N., Tanimura H., 2019, A\&A, 638, A75

Bykov A. M., Paerels F. B. S., Petrosian V., 2008, Space Sci. Rev., 134, 141

Cautun M., van de Weygaert R., Jones B. J. T., 2013, MNRAS, 429, 1286

Cautun M., van de Weygaert R., Jones B. J. T., Frenk C. S., 2014, MNRAS, 441, 2923

Cen R., Ostriker J. P., 1999, ApJ, 514, 1

Codis S., Pichon C., Devriendt J., Slyz A., Pogosyan D., Dubois Y., Sousbie T., 2012, MNRAS, 427, 3320

Codis S., Pichon C., Pogosyan D., 2015, MNRAS, 452, 3369

Colberg J. M., Krughoff K. S., Connolly A. J., 2005, MNRAS, 359, 272

Cornuault N., Lehnert M. D., Boulanger F., Guillard P., 2018, A\&A, 610, A75

Cui W., Knebe A., Yepes G., Yang X., Borgani S., Kang X., Power C., Staveley-Smith L., 2018a, MNRAS, 473, 68

Cui W. et al., 2018b, MNRAS, 480, 2898

Cui W. et al., 2019, MNRAS, 485, 2367

Danovich M., Dekel A., Hahn O., Teyssier R., 2012, MNRAS, 422, 1732

Davé R. et al., 2001, ApJ, 552, 473

Dekel A. et al., 2009, Nature, 457, 451

Dolag K., Meneghetti M., Moscardini L., Rasia E., Bonaldi A., 2006, MNRAS, 370, 656

Dubois Y., Pichon C., Haehnelt M., Kimm T., Slyz A., Devriendt J., Pogosyan D., 2012, MNRAS, 423, 3616

Eckert D. et al., 2015, Nature, 528, 105

Forero-Romero J. E., Hoffman Y., Gottlöber S., Klypin A., Yepes G., 2009, MNRAS, 396, 1815

Galárraga-Espinosa D., Aghanim N., Langer M., Gouin C., Malavasi N., 2020, A\&A, 641, A173

Ganeshaiah Veena P., Cautun M., Tempel E., van de Weygaert R., Frenk C. S., 2019, MNRAS, 487, 1607

Gheller C., Vazza F., 2019, MNRAS, 486, 981

Gheller C., Vazza F., Favre J., Brüggen M., 2015, MNRAS, 453, 1164

Gurbatov S. N., Saichev A. I., Shandarin S. F., 1989, MNRAS, 236, 385

Haggar R., Gray M. E., Pearce F. R., Knebe A., Cui W., Mostoghiu R., Yepes G., 2020, MNRAS, 492, 6074

Hamabata A., Oguri M., Nishimichi T., 2019, MNRAS, 489, 1344

Hidding J., Shandarin S. F., van de Weygaert R., 2014, MNRAS, 437, 3442

Klypin A., Yepes G., Gottlöber S., Prada F., Heß S., 2016, MNRAS, 457, 4340

Knollmann S. R., Knebe A., 2009, ApJS, 182, 608

Kraljic K. et al., 2018, MNRAS, 474, 547

Kraljic K. et al., 2019, MNRAS, 483, 3227

Kuchner U. et al., 2020, MNRAS, 494, 5473

Laigle C. et al., 2015, MNRAS, 446, 2744

Libeskind N. I., Hoffman Y., Knebe A., Steinmetz M., Gottlöber S., Metuki O., Yepes G., 2012, MNRAS, 421, L137

Libeskind N. I., Tempel E., Hoffman Y., Tully R. B., Courtois H., 2015, MNRAS, 453, L108

Libeskind N. I. et al., 2018, MNRAS, 473, 1195

Malavasi N., Aghanim N., Douspis M., Tanimura H., Bonjean V., 2020, A\&A, 642, A19

Malavasi N. et al., 2017, MNRAS, 465, 3817

Martínez H. J., Muriel H., Coenda V., 2016, MNRAS, 455, 127

Murante G., Monaco P., Giovalli M., Borgani S., Diaferio A., 2010, MNRAS, 405, 1491

Navarro J. F., Frenk C. S., White S. D. M., 1996, ApJ, 462, 563

Nicastro F. et al., 2013, ApJ, 769, 90

Oman K. A., Hudson M. J., Behroozi P. S., 2013, MNRAS, 431, 2307 
Paul S., Iapichino L., Miniati F., Bagchi J., Mannheim K., 2011, ApJ, 726, 17

Pereyra L. A., Sgró M. A., Merchán M. E., Stasyszyn F. A., Paz D. J., 2019, MNRAS , 499, 4876

Pichon C., Pogosyan D., Kimm T., Slyz A., Devriendt J., Dubois Y., 2011, MNRAS, 418, 2493

Power C. et al., 2020, MNRAS, 491, 3923

Rasia E. et al., 2015, ApJ, 813, L17

Reimers D., 2002, Space Sci. Rev., 100, 89

Rost A., Stasyszyn F., Pereyra L., Martínez H. J., 2020, MNRAS, 493, 1936

Shandarin S. F., Klypin A. A., 1984, Sov. Astron., 28, 491

Shandarin S. F., Zeldovich Y. B., 1989, Rev. Mod. Phys., 61, 185

Sousbie T., 2011, MNRAS, 414, 350

Sousbie T., Colombi S., Pichon C., 2009, MNRAS, 393, 457

Springel V., 2005, MNRAS, 364, 1105
Tanimura H., Aghanim N., Bonjean V., Malavasi N., Douspis M., 2019, A\&A, 637, 41

Tanimura H., Aghanim N., Bonjean V., Malavasi N., Douspis M., 2020, A\&A, 637, A41

Tempel E., Stoica R. S., Martínez V. J., Liivamägi L. J., Castellan G., Saar E., 2014, MNRAS, 438, 3465

Tempel E., Stoica R. S., Saar E., 2013, MNRAS, 428, 1827

Umehata H. et al., 2019, Science, 366, 97

Walker S. et al., 2019, Space Sci. Rev., 215, 7

Welker C. et al., 2020, MNRAS, 491, 2864

Zel'dovich Y. B., 1970, A\&A, 500, 13

This paper has been typeset from a $\mathrm{T}_{\mathrm{E}} \mathrm{X} / \mathrm{LAT}_{\mathrm{E}} \mathrm{X}$ file prepared by the author. 